\title{
Commentary \\ Using a global proteomic approach to identify proteins affected by estrogen therapy Timothy D Veenstra
}

Address: Laboratory of Proteomics and Analytical Technologies, Advanced Technology Program, SAIC-Frederick Inc., National Cancer Institute at Frederick, Frederick, MD 21702, USA. Email: veenstra@ncifcrf.gov

Published: 5 June 2009

Genome Medicine 2009, 1:58 (doi: 10.1 186/gm58)

The electronic version of this article is the complete one and can be found online at http://genomemedicine.com/content///6/58

(c) 2009 BioMed Central Ltd

\begin{abstract}
With the increase in technological capabilities for measuring biological molecules, there is a greater trend to conduct non-biased, discovery-driven studies that collect information on hundreds of molecules in a single study. The hope is that novel findings can be detected within these large datasets. For protein analysis, these non-biased studies are particularly challenging as no technology is presently capable of providing a view of the entire proteome. The ability of nonbiased studies to accurately detect specific differences within the proteomes of samples obtained from differentially treated individuals must be conclusively demonstrated before investigators will routinely adopt these methods as part of their experimental protocols. This need is especially true for clinical and epidemiological studies in which limited amounts of samples are available.
\end{abstract}

Conjugated equine estrogens (CEE) have been widely administered for the relief of menopausal symptoms [1]. While there are obvious benefits to women suffering from menopausal symptoms, estrogens are also known to exert harmful effects. For instance, the evidence linking estrogen metabolite levels and cancers of the reproductive system continues to grow [2]. Scientists are also studying the effects of estrogen metabolites in different racial populations. With this information, physicians now are faced with wondering which of their patients should have CEE administered. The short-term benefits need to be carefully weighed against any possible long-term detriments.

In 2003, the Women's Health Initiative (WHI) reported that women being administered CEE plus progesterone had an increased number of cardiovascular events compared to those given a placebo [3]. Unfortunately, confusion about the benefits of hormone replacement therapy (HRT) was introduced with the 2004 WHI estrogen-only trial that showed no increase in such cardiovascular events. Although this study did not provide a conclusive answer, it did suggest that women aged 50 to 59 years who received estrogen therapy enjoyed lower rates of heart disease [4]. A recent study, entitled the WHI Coronary-Artery Calcium Study (WHI-CACS), examined the effects of estrogen on coronaryartery calcification in women between the ages of 50 and 59 who were enrolled in the CEE trial [5]. The results of this study showed that women receiving estrogen had less coronary-artery calcification compared to the control group receiving a placebo.

Estrogen therapy is known to affect large numbers of proteins and metabolites, beyond those related to cardiovascular events. Low- and high-density lipoproteins, triglycerides, insulin, insulin-like growth factors, glucose and C-reactive protein are just a few of the molecules whose levels have been shown to be affected by estrogen administration. Considering the wide range of effects that estrogen exerts, there are undoubtedly many as yet undiscovered molecules and functional pathways that are affected by estrogen treatment. In the recent article published in Genome Medicine entitled 'Application of serum proteomics to the Women's Health Initiative conjugated estrogen equine estrogens trial reveals a multitude of effects relevant to 
clinical findings', Katayama et al. [6] sought to discover novel proteomic changes affected by CEE and also validate the efficacy of using a global proteomic strategy for finding these differences. This group used a method termed intact protein analysis system (IPAS) to compare protein levels in serum samples collected from women prior to initiation of CEE treatment and one year after initiation of treatment. Five separate analyses were conducted using samples acquired from 50 women. The samples were prepared by pooling equal-volume aliquots from groups of ten women. The IPAS system utilizes two dimensions of chromatography (anion exchange followed by reversed-phase) to fractionate intact proteins. Aliquots collected from the reversed-phase column are tryptically digested and the proteins are identified and quantified using high-throughput mass spectrometry (MS). Prior to their chromatographic separation, the proteins from the CEE-treated and control group were differentially isotopically labeled to allow their relative abundances to be measured via the MS measurements. All of the serum samples were also subjected to high-abundant protein depletion, eliminating such proteins as albumin and immunoglobulins from the analysis. The combination of high-abundant protein removal and extensive fractionation permitted proteins across seven orders of magnitude of abundance to be measured.

A total of 611 proteins were quantified between the serum samples acquired pre- and one-year post-CEE treatment. A statistically significant difference in the abundance of 116 proteins was observed between the two comparative samples. Of these, 64 proteins had a false discovery rate (FDR) less than 0.5 relative to all of the quantified proteins. Classification of these proteins into networks revealed five significant functional processes that were affected by CEE treatment: blood coagulation, kallikrein-kinin system, cell adhesion-platelet-endothelium-leukocyte interactions, complement system, and ossification. Comparison of these 64 proteins to previous findings yielded 13 (>20\%) that had been reported in the literature to have an association with estrogen therapy. The remaining 41 proteins where no association with estrogen therapy had been previously reported were associated with functions such as blood coagulation, ossification, cell growth, blood pressure maintenance, blood vessel morphogenesis, and angiogenesis. To confirm the IPAS results, enzyme-linked immunosorbent assays (ELISAs) were carried out on 13 of the proteins using both non-pooled samples that were part of the original IPAS study and an independent sample set. In both validation tests, the correlation between the results obtained from these two methods was $>0.83$.

This manuscript provides an excellent demonstration of the value of global comparative proteomic studies. Using a nonbiased method, Katayama et al. were able to correlate their results with a number of findings that had previously been reported to be associated with CEE. For example, CEE has been associated with a reduction in hip fractures. The authors were able to discover several proteins related to ossification and osteogenesis that had increased abundances in samples obtained from women that had been administered CEE. They were also able to show changes in several proteins that play roles in circulatory processes such as coagulation, angiogenesis, and blood pressure regulation. These results may be connected to the previously mentioned benefits of lower heart disease rates.

There is a misconception related to the value of the information to be gleaned from global proteomic studies as illustrated by Katayama et al. Many scientists that are unfamiliar with the technology anticipate that a global comparison provides a 'neat little package' of proteins whose abundance is affected by a particular stimulus. Unfortunately, many investigators are exasperated when the data they receive back is a long list of proteins with a large number of them showing a change in abundance. Often investigators find that the number of observed differences overwhelms their ability to decide which direction to follow. Katayama et al. have done an excellent job demonstrating the efficacy of turning a list of proteins into meaningful conclusions and further hypotheses. By grouping the proteins that showed a significant change in abundance they were able to uncover functional pathways that were affected by CEE treatment. These findings can now be used to interrogate specific proteins in order to gather a greater depth of information concerning the effect of CEE on a physiological function. In this study, a number of CEE-associated functions were uncovered, some previously shown and some novel. Following up on these non-biased results requires selection of those functions that are most critical from a public health view. This decision, while highly subjective, needs to carefully consider the quality of the data obtained in the global comparative study and the relative functional importance of each protein pathway. For example, in the search for a cancer biomarker, considerable effort would not be exerted to further study acute phase response proteins, although many of these would probably be detected as having higher abundances in samples taken from canceraffected individuals.

What this study has illustrated is the utility of global comparative proteomic analyses for identifying changes in protein abundances within clinical samples obtained from different groups of individuals. The affected proteins were adequately validated using an orthogonal method and independent sample set. While this study is not the first to use a non-biased global approach to discover proteins and molecules that change as a result of an external perturbation, health scientists and institutions are still reluctant to utilize technologies (such as the type used in this study) to conduct investigative research. While such global proteomic studies can be time consuming, taking months to complete, the richness of information obtainable more than makes up 
for the effort spent. In this CEE study, five functional groups of proteins were discovered to be perturbed, using the same data set. This wealth of information provides large numbers of hypotheses to be tested. Non-biased global studies, as illustrated in this manuscript, are a very efficient route to discovering novel effects of perturbations to biologic systems.

\section{Abbreviations}

CEE, conjugated equine estrogens; ELISA, enzyme-linked immunosorbent assay; FDR, false discovery rate; HRT, hormone replacement therapy; IPAS, intact protein analysis system; MS, mass spectrometry; WHI, Women's Health Initiative; WHI-CACS, WHI Coronary-Artery Calcium Study.

\section{Competing interests}

The author declares that he has no competing interests.

\section{Acknowledgements}

This project has been funded in whole or in part with federal funds from the National Cancer Institute, National Institutes of Health, under Contract HHSN26I20080000IE. The content of this publication does not necessarily reflect the views or policies of the Department of Health and Human Services, nor does mention of trade names, commercial products, or organizations imply endorsement by the US Government.

\section{References}

I. Nelson HD: Commonly used types of postmenopausal estrogen for treatment of hot flashes: scientific review. JAMA 2004, 291:1610. 1620.

2. Eliassen $\mathrm{AH}$, Hankinson SE: Endogenous hormone levels and risk of breast, endometrial and ovarian cancers: prospective studies. Adv Exp Med Biol 2008, 630: I48-I65.

3. Manson JE, Hsia J, Johnson KC, Rossouw JE, Assaf AR, Lasser NL, Trevisan M, Black HR, Heckbert SR, Detrano R, Strickland OL, Wong ND, Crouse JR, Stein E, Cushman M; Women's Health Initiative Investigators: Estrogen plus progestin and the risk of coronary heart disease. N Engl J Med 2003, 349:523-534

4. Anderson GL, Limacher M, Assaf AR, Bassford T, Beresford SA Black H, Bonds D, Brunner R, Brzyski R, Caan B, Chlebowski R, Curb D, Gass M, Hays J, Heiss G, Hendrix S, Howard BV, Hsia J, Hubbell A, Jackson R, Johnson KC, Judd $H$, Kotchen JM, Kuller L, LaCroix AZ, Lane D, Langer RD, Lasser N, Lewis CE, Manson J, et al:: Effects of conjugated equine estrogen in postmenopausal women with hysterectomy: the Women's Health Initiative randomized controlled trial. JAMA 2004, 291: I70I-I7I2.

5. Manson JE, Allison MA, Rossouw JE, Carr JJ, Langer RD, Hsia J, Kuller LH, Cochrane BB, Hunt JR, Ludlam SE, Pettinger MB, Gass M, Margolis KL, Nathan L, Ockene JK, Prentice RL, Robbins J, Stefanick $\mathrm{ML} ; \mathrm{WHI}$ and WHI-CACS Investigators: Estrogen therapy and coronary-artery calcification. N Engl J Med 2007, 356:259I-2602.

6. Katayama H, Paczesny S, Prentice R, Aragaki A, Faca VM, Pitteri S], Zhang Q, Wnag H, Silva M, Kennedy J, Rossouw J, Jackson R, Hsia J, Chlebowski R, Manson J, Hanash S: Application of serum proteomics to the Women's Health Initiative conjugated equine estrogens trial reveals a multitude of effects relevant to clinical findings. Genome Med 2009, 1:47. 